\title{
Der Zionismus und sein Popanz
}

Eine Antwort an die ,linken" Zionisten

\author{
Mosche Machover, Mario Offenberg
}

Acht Jahre sind seit dem Beginn der Besetzung der im Junikrieg 1967 eroberten Gebiete durch Israel vergangen. Die palästinensische Befreiungsbewegung hat einen großen Aufschwung genommen und wurde zu einem nicht mehr wegzudenkenden Faktor bei jeder Diskussion über die Perspektiven der Palästinafrage und des Nahostkonfliktes. Die relativen Erfolge der arabischen Armeen über Israel im Oktoberkrieg 1973, die wirtschaftliche und ideologische Brüchigkeit des israelischen Staates und schließlich die veränderte Haltung der USA und der westeuropäischen Staaten gegenüber den arabischen Staaten - mit der damit verbundenen zwangsläufig erfolgenden Nuancenverschiebung in der Frage der israelisch-arabischen Konfrontation - offenbaren überdeutlich die innen- und außenpolitische Schwächung der Position Israels. Die Isolienung Israels, die in der UNO symbolisch in den 104 Stimmen für die Palästina-Resolution zum Ausdruck gekommen ist, hat international gesehen nicht nur in den Ländern der III. Welt und Osteuropas, sondern in unterschiedlichem Maße auch in der westlichen Hemisphere stattgefunden: die Bundesrepublik ist hiervon nicht ausgenommen.

Während sich die westdeutschen bürgerlichen Massenmedien noch in Bekundungen von „Solidarität" und „Besorgnis" für das „,bedrohte“ Israel üben, aber auch erstmals - vorsichtig und verzerrt -, über den Kampf der Palästinenser für nationale Selbstbestimmung berichten, beurteilt die westdeutsche Linke den Nahostkonflikt im Zusammenhang mit ihrer antiimperialistischen Politik. Sie sieht die Ursachen des Nahostkonfliktes in der Tatsache, daß der Zionismus, eine reaktionäre, mit dem Imperialismus verbundene kolonisatorische Bewegung, ihr Vorhaben, nämlich die Schaffung des zionistischen Staates Israels, auf Kosten eines anderen Volkes verwirklicht hat. Nach seiner Gründung hat Israel die Rolle eines „Wachhundes" für die imperialistischen Interessen im arabischen Osten übernommen.

Es ist jedoch klar, daß der Zionismus und seine Helfer alles daran setzen um im Ausland durch propagandistische Tätigkeit, durch geschichtsklitternde Darstellungen und Appelieren an Emotionen den Abbauprozeß seiner Politik und Positionen aufzuhalten oder zurückzudrängen. Diese Propagandisten treten heutzutage nicht mehr in der althergebrachten Haltung des „150\% Rechthabenden schwachen Pioniers" auf und auch nicht in der Gestalt des arroganten Nationalisten, der für Groß-Israel und für die Vertreibung der Araber ist. Heute wird das subtiler und bescheidener gemacht, und dies hat seinen guten Grund: wo die reale politische und militärische Entwicklung im Nahen Osten, die Absichten eines multinationalen Palästina-Staates oder einer anderen nationalen Neunrdnung im Lande und die internationale Kräftekonstellation ernsthaft die Frage nach den zionistischen In- 
halten des israelischen Staates stellen, wird von prozionistischer Seite nun danach gestrebt, den Palästina-Konflikt in Begriffen einer ,tragischen Konfrontation zwischen zwei gleichermaßen berechtigten nationalen Aspirationen" hinzustellen der auf der Grundlage der Einfrierung der zionistischen Errungenschaften von 1949 (mit „Korrekturen“) beizulegen sei.

In diesem Aufsatz soll dargestellt werden, wie die objektiven und subjektiven Helfer des Zionismus in der Bundesrepublik, in ihrem Versuch die ZionismusKritiker zu verunsichern, eine „links"-gefärbte Polemik für den israelischen Staat und besondern gegen seine jüdischen Opponenten aus der antizionistisch-sozialistischen Bewegung Israels führen.

Vor einiger Zeit veröffentlichte die Zeitschrift „links“ aus Offenbach in einigen Fortsetzungen den Artikel ,Zum Klassencharakter der israelischen Gesellschaft", der im Jahre 1970 von Haim Hanegbi, Mosche Machover und Akiva Orr, Mitglieder der Israelischen Sozialistischen Organisation - „Matzpén“, geschrieben wurde (1). Ein Leser von ,links“, Alfred Moos wandte sich in einer Kritik sowohl gegen diesen ,Matzpén“ - Artikel als auch gegen die antizionistische Position von Matzpén überhaupt (2).

Der Artikel von Alfred Moos wird von uns als beispielhaft für die Argumentation sogenannter „linker" Zionisten angesehen. Wenn wir daher im folgenden auf die zentralen Punkte der Argumentation in diesem Artikel eingehen, wollen wir gieichzeitig versuchen, die Haltung ,linker" Zionisten insgesamt an diesem Beispiel zu erläutern und zu kritisieren und zu zeigen suchen, wie trotz aller Nuancen diese Position dennoch nahtlos in das Konzept der offiziellen zionistischen Propaganda paßt.

Zunächst jedoch eine Vorbemerkung:

Der Angriff gegen den „Matzpén“-Artikel nutzt die Tatsache aus, daß dieser keine historische Analyse des Zionismus enthält und zwar weder von der Seite des

1 Vgl. Haim Hanegbi, Mosche Machover, Akiva Orr, Zum Klassencharakter der israelischen Gesellschaft, in: links Nr. 30, 31, 32 (1972)

2 Vgl. Alf red Moos, in: links Nr.33 (1972).

Eine hebräische Fassung von Moos' Artikel wurde prompt in Israel von der zionistischen Gruppe, die sich 1965 von der KP Israels abgespalten hatte, die Maki, heute: Moked, in ihrem Organ 'Kol Ha'am' Nr. 32 (1972) unter dem Titel 'Der Popanz Zionismus' herausgebracht. Diese Gruppe hat es sich zur Aufgabe gemacht, dem israelischen Staat Schützenhilfe zu leisten, indem sie ,aus kommunistischer Sicht' alle Gegner der zionistischen Politik des Antisczialismus bezichtigt und sehr dankbar jeden politischen oder apologetischen Beitrag aus dem Ausland aufgreift. Sozialistische Positionen werden von diesen Leuten revidiert, nicht ausschließlich unter Anführung der klassischen zionistischen Erklärungen, sondern durch solche historizistische Konstruktionen, die die tatsächlichen Ereignisse und Fehlentwicklungen in der internationalen kommunistischen Bewegung und in der Sowjetunion für die von ihnen gewünschte Schlußfolgerung verwenden, die sozialistische Gegnerschaft zum Zionismus sei ja auch nur eine Fehlentwicklung mehr, die so ähnlich wie die ,Stalinisierung der SU und der Komintern zu verurteilen und rückgängig zu machen sei, wenn im folgenden von der Position ,linker Zionisten die Rede ist, beziehen wir uns auf diesen Artikel von A. Moos. 
Verhältnisses des Zionismus zur Judenfrage in Europa noch von der Seite des Verhältnisses des Zionistischen Unternehmens zu der Mehrheit der einheimischen Bevölkerung Palästinas (das arabisch-palästinensische Volk) und zu den verschiedenen imperialistischen Mächten, die in der Region seit dem Beginn der zionistischen Kolonisation und bis zum heutigen Tage herrschen.

Der Grund dafür, daß eine solche Analyse in jenem Artikel fehlt, ist einfach: Es war nicht die Absicht des Artikels, eine umfassende historische Abrechnung mit dem Zionismus zu liefern, sondern spezieller die Grundkonturen der heutigen israelischen Klassengesellschaft aufzuzeigen (3).

\section{Zionismus und Antisemitismus}

Es ist bezeichnend, daß ,linke“ Zionisten ihre Angriffe auf israelische Antizionisten immer wieder mit dem Hinweis beginnen, daß die jüdischen Einwanderer nach Palästina - die den menschlichen Rohstoff für das zionistische Unternehmen darstellten - ,allzu häufig vor der physischen Vernichtung, zumindest aber vor antisemitischer Erniedrigung und Existenznot geflohen sind“. Hinter dieser Einleitung verbirgt sich jene Drohung, welche die Propagandisten des Zionismus so sehr mögen und so oft verwenden: Wer den Zionismus anprangert, wer den Israelischen Staat ablehnt, wer gegen den zionistischen Charakter und die zionistische Politik Israels kämpft, ist ein Verbündeter des Antisemitismus.

Die Drohung kann auch in noch unverblümterer Weise ausgesprochen werden, wenn es z.B. heißt, daß der gegenwärtige Kampf gegen den Zionismus „, mit Krümeln aus der national-sozialistischen Küche geschmückt ist“. Und weiter: „Gelegentlich hat man fast den Eindruck, als ob die Zionisten für manche Linke die neu kostümierten ,Weisen von Zion' wären." Auch Worte der Warnung und der Drohung gibt es direkt an die Adresse antizionistischer Israelis: „Junge Israelis, die zum Kampf gegen den Zionismus aufrufen, sollten aber auch nicht vergessen, daß ihre Eltern oder Großeltern in weitaus meisten Fällen Verfolgte waren, für die Palästina/Israel die einzige Zufluchtstätte war und daß sie heute kaum das Recht hätten, die Grenzen Israels zu sperren, wenn irgendwann in der Zukunft Juden gezwungen sein

Die hebräische Originalfassung des genannten Matzpen-Artikels war ursprünglich im Tel-Aviver Organ ,Matzpen' erschienen und die Verfasser gingen von der Annahme aus, daß der Leser die Analysen der Organisation zur Geschichte und zum Wesen des Zionismus, wie sie in vielen Artikeln seit 1961 dargelegt wurden, kennt. Es ist klar, daß auch hier in unserer Antwort diese Analysen nicht im Detail wiederholt werden können. Teile davon sind abgedruckt in: Arie Bober (Hrsg.), The other Israel - The Radical Case against Zionism, New York 1972, vgl. auch Abdruck in den Heidelberger Zeitschriften: Neues Rotes Forum, Al-Djabha, Die Front; die Westberliner Zeitschrift, diskussion" Nr. 28/29 u. Nr. 30/31; SoPo Nr. 2 (1969) und Nathan Weinstock, Das Ende Israels? Berlin 1975.

Hier werden wir auf historische Fragen nur insofern eingehen, als es zur Widerlegung der Argumentation sog. ,Linker' zionistischer Kritik am Antizionismus erforderlich ist. 
sollten, sich vor antisemitischer Verfolgung nach Israel zu retten. Der alte jüdische Selbsthaß treibt manchmal seltsame neue Blüten".

Solche Verleumdungen sind nicht neu. Sie richteten sich schon gegen die jüdischen Kommunisten Russlands, die im II. Weltkongreß der Kommunistischen Internationale den Zionismus anprangerten:

„Es handelt sich um die Angelegenheit der Zionisten in Palästina, die unter dem Vorwand, einen unabhängigen israelitischen Staat zu gründen, die Arbeiterbevölkerung unterdrücken, die in Palästina lebenden Araber unter das Joch der Engländer zwingen, während die Israeliten doch nur in der Minderheit dort sind. Diese Lüge ohnegleichen muß bekämpft werden, und zwar in einer sehr energischen Weise, da die Zionisten in allen Ländern arbeiten, indem sie sich an alle rückständigen israelitischen Arbeitermassen heranmachen und Proletariergruppen mit zionistischen Tendenzen zu schaffen suchen (Poalei Zion), die in letzter Zeit bestrebt sind, eine kommunistische Phraseologie anzunehmen. (...) Die Kommunistische Internationale muß diese Bewegung aufs energischste bekämpfen“ (4). Einer der bekanntesten Repräsentanten des Zionismus machte aus seiner Meinung über die antizionistischen Kommunisten kein Hehl: „Diese vom $\mathrm{Haß}$ zu jeder jüdischen Sache durchsetzte Psychopathen und Sadisten werden in ihrer Verkommenheit und Hässlichkeit verderben und in ihrem eigenen Dreck ersticken“ (5). Der Umgang der Zionisten mit ihren (jüdischen) Kritikern, die auf den Prinzipien des Internationalismus gründend sie bekämpfen ist seither der gleiche geblieben. Der Mitbegründer der präkommunistischen Gruppe Palästinas wurde 1920 zum „Verräter" uñ zum „Feind des jüdischen Volkes" abgestempelt (6), weil er sich im Ausland zu sagen erlaubte, daß die Vertreibung der arabischen Fellachen durch die Zionistische Bewegung die ganze arabische Welt gegen die Juden Palästinas herausfordert (7). Sogar die „Tauben“ des Zionismus kennen da kein Pardon; für sie leiden die Antizionisten aus dem ,heiligen Land“ unter einer „,krankhaften Feindseligkeit dem nationaljüdischen Neuentstehungswerk gegenüber", da sie den „Glauben der Aufhetzung zum Ausrottungskrieg gegen die jüdische Gemeinschaft des Landes“ verbreiten (8).

Die israelischen revolutionären Sozialisten sind an den Vorwurf des „Selbsthasses" seit jeher gewohnt, und gegen ihn gut gewappnet. Jedoch wissen sie auș eigener Erfahrung, daß dieser Popanz der diffamierenden Identifizierung „Antizio-

$4 \quad$ Rede von Esther Maria Frumkina, in: Der 2. Kongreß der Kommunistischen Internationale. Prot. der Verhandlungen vom 19. 7. in Petrograd und vom 23. 7. bis 7. 8. 1920 in Moskau, hrsg. vom Verlag der KI, Hamburg 1921 S. 198

5 David Ben Gurion, Memoiren Teil I, Tel Aviv 1971, S. 245 (hebr.)

6 vgl. z.B. in: Kontres, Organ der Achduth Ha'awoda, Nr. 47, Tel Aviv 1920 (hebr.)

7 Yaakow Meiersohn, Nach der 5. Poalei-Zion-Konferenz - Brief an die Genossen der Sozialistischen Arbeiterpartei in Palästina (jiddisch), Wien 1920, abgedruckt in: Mario Offenberg, Vom Zionismus zum Internationalismus. Die Entstehungsgeschichte der Kommunistischen Partei Palästinas (P.K.P.) 1921 - 1925, Phil. Diss. Berlin 1974 (MS), demnächst gedruckt: Meisenheim 1975

8 Aharon Cohen, Israel und die arabische Welt, Tel Aviv 1964, S. 259 (hebr.) 
nismus $=$ Antisemitismus" noch einen beachtlichen Teil der Linken (geschweige denn der demokratischen Nicht-Linken) außerhalb Israels einzuschüchtern vermag. Aus verständlichen Gründen ist die westdeutsche Linke dieser zionistischen Verleumdung gegenüber besonders empfindlich. Es ist daher unerläßlich, daß die Linke auch in Westeuropa lernt, diese falsche und diffamierende Gleichsetzung ats propagandistischen Popanz zionistischer Politik zu durchschauen.

Es unterliegt keinem Zweifel, daß die moderne zionistische Bewegung als eine Reaktion auf den Antisemitismus und auf die schwere Lage der Juden in Ost- und Zentraleuropa am Ende des 19. und zu Beginn dieses Jahrhunderts entstand. Aber es genïgt nicht festzustellen, daß der Zionismus eine Reaktion auf den Antisemitismus darstellt; wir müssen feststellen, welcbe Art Reaktion dies ist. Prinzipiell kann es gegenüber dem Antisemitismus wie auch gegen anderen ähnlichen Erscheinungen der Diskriminierung und der Unterdrückung aus rassischen, ethnischen, religiösen und ähnlichen Gründen zwei entgegengesetzte Einstellungen geben.

Die erste Einstellung ist Gemeingut nicht nur der Sozialisten, sondern auch aller derjenigen, die eine fortschrittliche Überzeugung haben (Radikal-Liberalen, Radikal-Demokraten usw.). Für sie entspringen die Erscheinungen der Diskriminierung und Unterdrückung von Minderheiten nicht dem menschlichen Charakter, vielmehr sind sie das Ergebnis von bestimmten Verhältnissen - namentlich von gesellschaftlichen, ökonomischen und politischen Verhältnissen, die also bistoriscb und somit auch veränderbar sind.

Diese Auffassung besagt, daß die politisch richtige Reaktion auf den Antisemitismus sowie auf andere ähnliche Erscheinungen allein in jenem Kampf zur Veränderung der bestehenden gesellschaftlichen, ökonomischen und politischen Verhältnisse besteht, welche ein organischer Bestandteil des allgemeinen Kampfes zur, sagen wir, „Weltverbesserung" ist. Gewiß unterscheiden sich verschiedene fortschrittliche Richtungen (revolutionäre Sozialisten, Sozialreformisten, Radikale) erheblich voneinander, sowohl in ihren Zielvorstellungen von der Neuen Welt zu der der allgemeine Kampf für den Fortschritt führen muß, als auch in den zur Führung des Kampfes notwendigen Mitteln. Aber alle teilen eine gemeinsame fundamentale Voraussetzung: Der Kampf gegen die Wurzeln des Antisemitismus und ähnlicher Erscheinungen ist sinnvoll und dieser Kampf (als Teil des allgemeinen Kampfes für eine bessere Gesellschaft) stellt die einzig richtige politische Antwort dar.

Demgegenüber finden wir bei denjenigen, die verschiedene reaktionäre und rassistische Anschauungen vertreten, im allgemeinen eine entgegengesetzte Einstellung: Der Gegensatz und der Konflikt zwischen der Mehrheit einer Bevölkerung und ihren rassischen, ethnischen oder religiösen Minderheiten, wurzelt in der "menschlichen Natur" selbst: Ein Kampf gegen den Antisemitismus (oder gegen ähnliche Erscheinungen) sei sinnlos, weil der Antisemitismus eine notwendige, normale ja sogar gesunde Erscheinung ist. Der einzige Weg, das Problem aus der Welt zu schaffen, liegt in der Vernichtung seiner angeblichen Wurzeln: Es gilt jenen Zustand zu verändern, in dem Juden als eine Minderheit inmitten von Nichtjuden leben. Es wird dem Leser leichtfallen, zu erkennen, daß diese zweite Einstellung für den Antisemiten charakteristisch ist. Jedoch die ganze Wahrheit ist, daß diese Ein- 
stellung die fundamentale Voraussetzung und den Ausgangspunkt darsteilt, sowobl für den Antisemitismus wie für den Zionismus. Der Unterschied besteht nur darin, daß der Zionismus die Juden dazu aufruft, selbst die „nichtjüdischen“ Völker zu verlassen, während der Antisemitismus einfach dazu auffordert, sie rauszuwerfen.

Man kann konstatieren, daß die Gemeinsamkeiten in den Grundlagen des Antisemitismus und des Zionismus vielen Antisemiten bewußt sind. So zum Beispiel verrät uns der britische Colonel R. Meinertzhagen (der als politischer Offizier im Stab des Eroberers Palästinas im 1. Weltkrieg, General Allenby, tätig war): „Allgemein gesehen ist meine Einstellung den Juden gegenüber durch einen antisemitischen Instinkt bestimmt, der durch den persönlichen Kontakt immer eine Veränderung erfährt. Meine Ansichten über den Zionismus sind die eines begeisterten Zionisten" (9).

Dieses freundliche Zuwinken des Antisemiten beantwortet der Zionist mit einer eleganten Verbeugung. In seinem Tagebuch erzähit der Begründer des Zionismus, Theodor Herzl, wie ihn der Dreyfuss-Prozeß beeinflußte, über den er, Herzl, für eine antisemitische Wiener Zeitung berichtete:

„In Paris (...) gewann ich ein freies Verhältnis zum Antisemitismus, den ich historisch zu verstehen und $\mathrm{zu}$ entschuldigen anfing. Vor allem erkannte ich die Leere und Nutzlosigkeit der Bestrebungen zur $A b w e b r$ des Antisemitismus" (10).

Die Ideologie des Zionismus, so wie sie von ihrem Begründer Theodor Herzl konzipiert wurde, gründet auf Vorarbeiten, die andere „Rassentheoretiker" leisteten. Für einen von ihnen unterliegt der Antisemitismus einem biologischen Gesetz: „Judeophobie ist eine Art Dämonopathie mit dem Unterschied, daß sie nicht Eigenschaft einer besonderen Rasse ist, sondern Gemeingut der ganzen Menschheit... Wie ein psychisches Gebrechen ist sie vererbbar und als Krankheit für zweitausend Jahre unheilbar".

Ein anderer „,Theoretiker“ in ,Sachen Juden“ sagt: „Die jüdischen Nasen werden nicht reformiert, und das schwarze, krause jüdische Haar wird durch keine Taufe in blondes, durch keinen Kamm in schlichtes verwandelt. Die jüdische Rasse ist eine ursprüngliche, die sich trotz klimatischer Einflüsse in ihrer Integrität reproduziert. Der jüdische Typus ist in sich im Laufe der Jahrhunderte stets gleich geblieben. (. . .) Den Juden und Jüdinnen hilft (es) nichts, durch Taufen und Untertauchen in das große Meer der indogermanischen und mongolischen Stämme ihre Abstammung zu verleugnen. Der jüdische Typus ist unvertilgbar". Obwohl diese Aussagen durchaus von der Schule Alfred Rosenbergs hätten stammen können, müssen wir die eigentlichen Verfasser nennen: der erste ist der zionistische Soziologe Leo Pinsker, der Zweite ist Moses Hess (11).

Es fällt nicht schwer, viele weitere Zitate aus den zionistischen Schriften anzuführen, von der Anfangszeit bis zum heutigen Tage, die die Gemeinsamkeit des

9 R. Meinerzhagen, Middle East Diary, London 1959, S. 49

10 T. Herzl, Tagebücher, Bd. I., Berlin 1922, S. 6/7

11 Leo Pinsker, Auto-Emancipation, New York 1948, S. 33 und M. Hess, Rom und Jerusalem, Tel Aviv 1935, S. 25/26 
theoretischen Ausgangspunktes von Zionismus und Antisemitismus beweisen. Wir werden dem Leser diese Zitate ersparen und uns mit der Analyse eines jungen zeitgenössischen israelischen Historikers, Yigal Elam, begnügen:

„Der Zionismus setzte den Antisemitismus als einen natürlichen Zustand in der Einstellung der Welt gegenüber den Juden voraus. (...) Der Zionismus sah im Antisemitismus keine anormale, absurde und perverse Randerscheinung. Der Zionismus betrachtete den Antisemitismus als eine Naturgegebenheit. Eine bis zur Norm befestigte Konstante in dem Verhältnis der Nichtjuden zur Anwesenheit der Juden in ihrer Mitte (...) Der Zionismus betrachtete den Antisemitismus als eine normale, beinahe rationale Reaktion der Nichtjuden auf einen nicht-normalen, absurden und perversen Zustand des juidischen Volkes in der Diaspora“ (12).

Entlarvend und aufschlußreich das beinahe entschuldigende Verständnis, das 1934 ein prominenter zionistischer Führer gegenüber dem National-Sozialismus zeigt:

„Aus den letzten Schlupfwinkel der Taufe und der Mischehe sind (die Juden) hervorgezogen worden. Wir sind nicht unglücklich darüber. Wir sehen in diesem Zwang zum Bekennen, zum klaren, eindeutigen Mut, zu seiner Gemeinschaft zu stehen, zugleich die Erfüllung unserer Sehnsüchte. (. . .) Die Theorie der Assimilation ist zusammengebrochen. Kein Schlupfwinkel birkt uns mehr. Wir wünschen an die Stelle der Assimilation das Neue gesetzt: das Bekenntnis zur jüdischen Nation und zur jüdiscben Rasse. Ein Staat, der aufgebaut ist auf dem Prinzip der Reinheit von Nation und Rasse (gemeint ist das Dritte Reich, A.d.V.), kann nur vor dem Juden Achtung und Respekt haben, der sich zur eigenen Art bekennt" (13).

Das weitgehende Einvernehmen zwischen Zionismus und Antisemitismus, bedingt durch die gemeinsamen ideologischen Ausgangspositionen, geht weiter als man es annehmen könnte...

In der Einleitung zu den berüchtigten Nürnberger Gesetzen vom 15.September 1935 wird u.a. gesagt: „Hätten die Juden bereits einen eigenen Staat indem die Masse ihres Volkes zuhause wäre, so könnte die Judenfrage schon heute als gelöst gelten, auch für die Juden selbst. Gerade von den überzeugten Zionisten ist desbalb am wenigsten Widerspruch gegen die Grundgedanken der Nürnberger Gesetze erboben worden, weil sie einmal wissen, daß diese Gesetze auch für das jüdische Volk die einzig richtige Lösung darstellen (...)“"(14).

Ein solches unausgesprochenes Einvernehmen zwischen Zionismus und Antisemitismus mußte eine knallende Ohrfeige für diejenigen Juden und Nicht-Juden

vgl. Y. Elam in einem Artikel in ,Ot', Organ der israelischen Arbeiterpartei Ma'arach Nr. 2, Tel Aviv 1967 (hebr.)

13 Das Zitat stammt aus einem Buch, das 1934 in Berlin erschien. Der Verfasser war damals einer der führenden Zionisten in Deutschland und ist heute ein führender Zionist in den USA und gleichzeitig Vorsitzender der internationalen Leitung des - zionistisch gelenkten - Jüdischen Weltkongresses.

vgl. J. Prinz, Wir Juden, Berlin 1934, S. 154 (im Original gesperrt!) 
sein, die die Lösung der Frage im politischen Kampf für die Demokratisierung ihrer Gesellschaften sahen. Isaac Deutscher berichtet, daß in Osteuropa und besonders in Polen, gerade die jiddisch sprechenden Arbeiter, die sich ohne Abstriche als Juden betrachteten, die entschiedensten Feinde des Zionismus waren. Sie waren entschlossene Opponenten einer Auswanderung nach Palästina. In der Idee einer Evakuierung, eines Exodus' aus den Ländern, in denen sie ihre Heimat hatten und ihre Vorfahren seit Jahrhunderten gelebt hatten, sahen diese Antizionisten einen Verzicht auf ihre Rechte, ein Nachgeben gegenüber einem feindseligen Druck, ein Verrat an ihrem Kampf und eine Förderung des Antisemitismus. Für sie schien der Antisemitismus im Zionismus zu triumphieren, der die Legitimation und Gültigkeit des alten Geschreis ,Juden raus" anerkannte. Die Zionisten akzeptierten es, sie wollten ,raus“" (15).

Der Zionismus ist zwar eine Reaktion auf den Antisemitismus; jedoch die Grundvoraussetzung auf der die zionistische Ideologie gründet, stimmt mit der des Antisemitismus überein.

\section{Zionismus und die Recbte der Juden}

Aus dem oben Ausgeführten wird klar, warum dem Zionismus der Kampf gegen Antisemitismus sowie für die Gleichberechtigung der Juden so oft gleichgültig war, da er die Möglichkeit und Nützlichkeit eines Kampfes gegen den Antisemitismus von vornherein bestreitet. Die Lage der außerhalb Palästinas lebenden Juden interessiert den Zionismus nur insofern, als die Juden durch ihre Lage bewegt werden, nach Palästína auszuwandern oder zumindest den Zionismus zu unterstïtzen. Dies wird vom israelischen Historiker Y. Elam, den wir bereits oben zitierten, folgendermaßen ausgedrückt: „Vom ersten Moment an sagte er (der Zionismus) sich von Überlegungen los, die mit der Lage des jüdischen Volkes in der Diaspora in Zusammenhang stehen; es sei denn, diese trügen zum zionistischen Unternehmen bei“. Und so kam es, ,daß in den Jahren nach der national-sozialistischen Machtergreifung in Deutschland, als in der ganzen jüdischen Welt die Demonstrationen und Protestaktionen gegen das NS-Terror-Regime ihren Höhepunkt erreichten, die Stimme des Zionismus nicht zu hören war" (16).

Die Zionisten in ihrer Gesamtheit lehnen das Weiterbestehen der „Diaspora“ ab; diese Einstellung betrachtet das Leben von Juden außerhalb Palästinas/Israels als verwerflich, während nur die Einwanderung dorthin, die aktive Beteiligung an das Zionistische Unternehmen als ,erstrebenswert" betrachtet wird. Bezüglich der Frage der Einstellung der Zionisten zu den in der Welt lebenden Juden sagt der

16 Y. Elam, Einführung in eine andere zionistische Geschichte, Tel Aviv 1972, S. 113 u. S. 122 (hebr.) 
israelische Geschichtsprofessor und langjähriger Zionistenfunktionär Arieh Tartakower: ,Sie (die Mehrheit im Zionismus) hielten jede Aktivität zum Schutz jüdischer Rechte in der Diaspora für völlige Kraftverschwendung" (17). Auch wenn aus zionistischer Sicht die Verachtung der „Diaspora“ ein scheinbarer Widerspruch war - da es ein aus eigennützigen Interessen doch nicht gleichgïltig sein könnte, was mit dem Einwanderer-Reservoir passiert -, scheint es doch so zu sein, daß die Zionisten (so wie ursprünglich Herzl) bis zu einem gewissen Punkt antisemitische Umtriebe, die die Juden zur Emigration nach Palästina bewegen könnten, für wichtiger hielten als die Bekämpfung des Antisemitismus. Zweifelsohne beinhaltet dieser Gedankengang in gewissem Maße ein Disziplinierungselement, zugleich eine Selbstbestätigung, in jedem Fall eine tiefe Menschenverachtung und eine grenzenlose Heuchelei.

Einzelne Zionisten wie Nahum Goldmann und Jitzhak Grienbaum forderten vor und während des II. Weltkrieges eine Beteiligung im Kampf um die Rechte der Juden. Jedoch alle Strömungen und alle wichtigen Führer des Zionismus wiesen diese Forderung zurück. Im Jahre 1935 setzte der Vorstand der „Jewish Agency“, die Institution, die die zionistischen Aktivitäten in Palästina leitete, eine besondere Kommission, für die Probleme der Juden in Deutschland ein. So kam es, daß in der Sitzung des Vorstandes der „Jewish Agency“ am 31. Dezember 1935, David Ben Gurion, in Beantwortung der Forderung von J. Grienbaum, die zionistische Bewegung solle am Kampf für die Rechte der Juden in Deutschland teilnehmen, feststellte, daß ,dies nicht die Aufgabe der Kommission (sei) die vom Vorstand zur Behandlung der Rechte der Juden in Deutschland nomminiert wurde. Die Aufgabe dieser Kommission war es, die Frage der Juden Deutschlands allein vom Aspekt ihrer Einwanderung nach Palästina zu beraten, und ihre Schlußfolgerungen stehen keineswegs im Widerspruch zu irgendwelchen Maßnahmen, die man für die Rechte der Juden Deutschlands ergreifen kann. Die Aufgabe der Kommission war es, den zionistischen Aspekt der Frage und nicht über Maßnahmen für die Rechte der Juden in der Diaspora zu beraten" (18)

Auch wenn wir akzeptieren, daß die Schlußfolgerungen dieser Kommission tatsächlich nicht im Widersprucb zu einem Kampf für die Rechte der Deutschen Juden standen (und schon dies ist keineswegs sicher!) so bleibt doch noch die Tatsache, daß die Kommission keineswegs willens war, sich um diesen Kampf zu kümmern. In der Tat war es die Hauptaufgabe dieser Kommission, die berühmte „Transfer"-Angelegenheit zu organisieren; jenen Handelsvertrag zwischen der Zionistischen Bewegung und der Regierung Hitlers, nach dem die Gelder und der Besitz deutscher Juden nach Palästina in Form von deutschen Waren überbracht wurden. Auch hier war es (wie Y. Elam mit Recht bemerkt) ,nicht das Bestreben, jüdischen Besitz in der Diaspora zu retten, das im Hintergrund des Vertrages stand, York 1954, S. 63 
sondern das Bestreben, die wirtschaftliche Kraft des jüdischen ,Yischuws in Palästina zu stärken" (19).

Diese Gleichgültigkeit des Zionismus gegenüber dem Kampf um die Rechte der Juden bestand seit jeher. Auch heute kommt diese Gleichgültigkeit z.B. in der Angelegenheit der sowjetischen Juden zum Ausdruck. Es muß darauf hingewiesen werden, daß das Ziel der lautstarken Kampagne die die Zionistische Bewegung in dieser Sache führt, nicht die Erlangung voller und gleicher Rechte für die Juden der Sowjetunion ist, sondern nur die Erlangung eines einzigen Recbtes, nämlich das Recht auf Auswanderung nach Israel. Der Kampf für die Rechte der sowjetischen Juden, der genauso wie jeder andere Kampf um die Erlangung von Rechten für eine nationale oder ethnische Minderheit ein Kampf ist, der die Unterstiitzung eines jeden fortschrittlichen Menschen verdient, interessiert den Zionismus kaum. Mehr noch: Wie wir später sehen werden, ist es nicht übertrieben anzunehmen, daß wenn der Auswanderungswille sowjetischer Juden -- aus welchen Gründen auch immer abnimmt, dies bei vielen zionistischen Führern Enttäuschung und Bedauern hervorruft.

Das Problem der juidischen Minderheit in der Sowjetunion besteht darin, daß die staatlichen und gemeinschaftlichen Einrichtungen im kulturellen sowie im religiösen dieser Minderheit nicht in dem Maße gewährleistet werden, wie es ihr Status als staatlich anerkannte Nationalitäten-Minorität, von der Verfassung her vorsieht. Auch wenn dies keine Entschuldigung sein kann für staatliche Versäumnisse muß es offen gelassen werden, wie groß derjenige Teil der jüdischen Sowjetbevölkerung ist, der tatsächlich Wert auf nationale Einrichtungen legt.

Jeder Versuch, dem ,jüdischen Problem“" in der S.U. die ahistorische ,ewige Dimension“ anhängen zu wollen - die dem Idealismus im allgemeinen und dem Zionismus insbesondere typisch ist -, ist von Anfang an manipulativ und irreführend und basiert hauptsächlich auf dem Mißbrauch der Emotionen und der Unkenntnis des Betrachters. Das ,jüdische Problem“ in der S.U. ist eins der vielen nationalen Probleme der S.U.; nicht das einzige, nicht das wichtigste; es besteht nicht ,autonom“ (nach dem Motto: ,sogar der Sozialismus kann nicht die Judenfrage lösen ..."), getrennt oder unabhängig von den anderen innersowjetischen gesellschaftlichen Prozessen und es kann auch nicht gelöst werden, ohne daß eine Lösung für die anderen innersowjetischen Probleme, die die sowjetische Gesellschaft beschäftigen, gefunden wird.

Es wäre sicherlich sehr vermessen den Auswanderungswillen sowjetischer Juden nur auf den Wunsch dieser Menschen zurückzuführen, umfassender als es in der S.U. möglich ist, jüdische religiöse und kulturelle Bedürfnisse zu befriedigen oder/und in Israel den Zionismus politisch, wirtschaftlich und militärisch zu stärken. Für einen Teil von ihnen mag das stimmen. Für viele aber steht im Vordergrund der Wunsch, außerhalb der Sowjetunion leben zu können. Ein Drittel der auswandernden beherrschte jüdische Gemeinschaft Paläsrinas vor 1948 
Sowjetjuden, deren Ziel Israel sein soll kommt dort nie an, sie springen schon während des Zwischenaufenthalts in Wien oder Rom von der „Reise nach Jerusalem" ab (20). Der russische Zionistenaktivist Dr. Viktor Polski, der Ende Dezember 1974 aus Moskau kommend in Israel einwanderte lamentiert: ,Sollten die Ausreisebedingungen gelockert und weniger ablehnende Bescheide von der Sowjetregierung erteilt werden habe ich gar keinen Zweifel, daß der Auswanderungsstrom erheblich zunehmen wird. Meine große Befürchtung ist aber, daß der Strom derer die in Israel eintreffen nicht proportionell anwachsen wird. Wenn sich das Israel-Image der Sowjetjuden und die realen Bedingungen die es erzeugen nicht ändern werden, wird der Anteil derer die auf dem Weg abspringen größer sein als derer die in Israel ankommen" (21). Womit innerisraelische Aspekte angesprochen sind.

Viele der sowjetischen Israel-Auswanderer sind der israelischen Propaganda, die durch Rundfunk und andere viel subtilere und scheinbar „unoffiziellen" Mitteln bis ins Tiefe der Sowjetunion hinein reichen, zum Opfer gefallen. Seit einiger Zeit hat sich die Situation zu verändern begonnen: Bereits ausgewanderte Verwandte und Bekannte geben ausführliche Berichte über das böse Erwachen, das sie im zionistischen Staat erlebten. Anstatt einer völlig harmonischen und konfliktlosen „Überflußgesellschaft" haben sie eine Klassengesellschaft vorgefunden, in der sie trotz der großen finanziellen Vergünstigungen die sie als bevorzugte Einwanderer genießen, der gleichen Ausbeutung, Arbeitslosigkeit, Inflation, Bürokratie, Entfremdung ausgesetzt sind, die das tägliche Leben der übrigen werktätigen Bevölkerung Israels ausmacht; hinzu kommt die ständige Todesgefahr durch die Konfrontation mit der arabischen Umwelt. Im Jahre 1974 sind halb soviel Juden aus der S.U. nach Israel ausgewandert wie jeweils in den vorangegangenen Jahren 1973 und 1972 (22). Mit der Verschärfung der Wirtschaftskrise in Israel, mit der steigenden Inflations- und Arbeitslosenraten wird der Unmut der israelischen Bevölkerung gegenüber der in Steuererleichterungen, Wohnung und Arbeitsplätze zunächst privilegierten Sowjetjuden stärker und entschiedener. Jeder der einheimischen Werktätigen kann sich leicht ausrechnen, daß der ohnehin unzureichende Kuchen des Nationaleinkommens und der von der Zionistischen Organisation im Ausland gesammelten Kapitalien noch ungerechter aufgeteilt werden würde. Konnte man in der Vergangenheit scheue Klagen hinter vorgehaltener Hand hören gegen die Bevorzugung der Einwanderer die „man“ aber brauche, so kann man heutzutage feststellen, daß die Proteste derer, die ihren Unmut öffentlich in Israel bekunden dürfen, also die Juden und konkret die benachteiligten Schichten unter ihnen, wie die Orientalen, Teile der Jugend und der Arbeiterschaft, gegen eine auf ihre Kosten gehende Einwanderung, klarer und lauter werden. Die Mehrheit von ihnen reagiert spontan, ohne sich im allgemeinen zugleich bewußt zu werden, daß sie bereits an 
einem der Grundprinzipien des Zionismus rütteln. Die Zusammenführung der Juden in Palästina/Israel, die demographische Majorisierung der Araber, die Fütterung mit menschlichem Rohstoff der unersättlichen - und langfristig doch unzureichenden - israelischen Militärmaschine für ihren Kampf aufs Ganze - das und einiges mehr ist Zionismus. Alle Einwanderungen nach Israel sind heute und in der Vergangenheit zionistisch bedingt, kontrolliert und eingesetzt. Der objektive Widerspruch zwischen der zionistischen Einwanderung und den Interessen der Werktätigen Israels ist nicht lösbar; er bleibt bestehen als eine zusätzliche Quelle innerisraelischer Klassenauseinandersetzungen (23).

Was geschieht aber mit den Israel-,,Abtrünnigen“?

Der israelische Journalist Abraham Tirosch berichtet über jüdische Auswanderer aus der S.U., die entweder in Israel angekommen sind und dieses Land verlassen haben oder die auf dem Wege von Moskau nach Tel Aviv - und trotz ziemlich strenger israelischer Bewachung - in Wien ,,abspringen“ können. (24) Diese Juden, die sich in einer Notlage befinden und die dringend Hilfe brauchen, werden von der sog. Organisation „der Juden“, die zionistische „Jewish Agency“, die in allen wichtigen westeuropäischen Städten Büros unterhält, in der Regel abgewiesen. In Rom befindet sich das europäische Büro der einzigen - angeblich - unabhängigen jüdischen Flüchtlingsorganisation, die „Hias". Tirosch berichtet weiter: Mittellos und desorientiert schleppen sich nun diese jüdischen Flüchtlinge nach Rom und Wien. „Die Hias-Organisation weigert sich, die sowjetischen Emigranten zu betreuen, die zu ihren Büros in Wien, Rom oder Israel gelangen, wenn sie nicht die Bestätigung und die Zustimmung der Jewish Agency, die jeden Fall eingehend prüft, erhalten hat. Der stellvertretende Direktor der Einwanderungsabteilung der Jewish Agency Yehuda Dominitz und leitende Kreise der Hias haben die Nachrichten, die in letzter Zeit verbreitet wurden, heftig dementiert, nach denen, im Gegensatz zum Abkommen, die Hias begonnen hätte, die sowjetischen Auswanderer aus Israel nach Europa und U.S.A. zu betreuen."

Als Fazit bleibt nach der Betrachtung des Problems der sowjetischen Juden: Die Zionistische Bewegung kämpft nicht für die Anerkennung des Rechtes eines jeden Menscben von einem Land in ein anderes auswandern zu können - an sich eine allgemein fortschrittliche Forderung die von jeden Sozialisten unterstützt werden sollte -, sondern sie fordert dieses Recht als Sonderprivileg lediglich für Juden, und auch hier nur unter der Bedingung, daß sie nach Israel und nicht in ein anderes Land emigrieren.

Die Basis für die zionistische Kampagne in der Angelegenheit der sowjetischen Juden ist nicht der allgemeine Gedanke der universellen Menschenrechte, sondern die zionistische These, nach der jeder Jude überall in der Welt ein besonderes politisches Recht auf Palästina hat. Und dies, während der Zionismus jegliche politi1973 , S. $16-25$ 
schen und nationalen Rechte der Araber Palästinas auf ihr Heimatland leugnet.

In der Tat, diese gleiche zionistische Regierung und diese gleiche zionistische Anschauung fordern für einen in Moskau geborenen Juden ein automatisches Recht, von der Sowjetunion nach Israel zu emigrieren und verleiht ihm automatisch die israelische Staatsangehörigkeit. Gleichzeitig negiert dieselbe Anschauung und dieselbe Regierung das Recht eines in Haifa geborenen Arabers, der heute zum Beispiel im Gazastreifen oder im Lager am Rande Beiruts als Flüchtling haust, in seine Heimatstadt zurückzukehren und dort seine Bürgerrechte zu erhalten.

Den Zionismus interessieren die allgemeinen Menschenrechte und auch die Rechte der Juden im allgemeinen nur soweit sie die Auswanderungen nach Israel fördern helfen.

Der ,grausame Zionismus"

Oben erwähnten wir bereits den Transfer, jenes moralisch zweifelhafte Geschäft zwischen der Zionistischen Bewegung und der Regierung Hitlers. Als dieses Geschäft kritisiert wurde (das war die Zeit, als fortschrittliche Kräfte zu einem wirtschaftlichen Boykott des Dritten Reiches aufriefen) erwiderte Mosche Schertok (später bekannt als M. Scharett, ein bekannter zionistischer Führer, und der erste Außenminister Israels) mit diesen Worten: „In diesem Punkt besteht ein Widerspruch zwischen der Diaspora und Eretz-Israel (d.h. das zionistische Unternehmen in Palästina, Anm. d.V.) ... Es ist das Schicksal des Zionismus, daß er der Diaspora gegenüber manchmal auch grausam sein muß, und zwar wenn der Aufbau des Landes dies erfordert" (25).

Diese Grausamkeit des Zionismus gegenüber den Juden in der Welt nimmt zuweilen besonders zynische Züge an. Es kann viele Menschen geben, die zu einer unterdrückten Gruppe gehören, die aber dennoch am Kampf gegen die Ursachen der Unterdrückung nicht teilnehmen wollen oder können und eine individuelle Lösung - durch Emigration in ein anderes Land - vorziehen. Die Sozialisten schlagen nicht vor, ihnen diese Möglichkeit zu nehmen; im Gegenteil: Sie bestehen auf dem Recht eines jeden Menschen auf freie Emigration; sie wenden sich aber entschieden dagegen, daß die Emigration als eine kollektive politiscbe Lösung präsentiert wird, die den Kampf gegen die Unterdrückung ersetzen soll. Es sei an dieser Stelle bemerkt, daß in den $20 \mathrm{er}, 30 \mathrm{er}$ Jahren und auch später viele der osteuropäischen Juden in der Tat diese individuelle Lösung der Emigration wählten. Viele Millionen wanderten aus den Ländern, in denen sie in Bedrängnis lebten, in die USA und in andere Länder aus, und praktisch lösten sie für sich zufriedenstellend ihr Problem. Die zionistische Auswanderung nach Palästina war im Vergleich zum Strom der 
jüdischen nicht-zionistischen Emigration nach anderen Ländern geringfügig. Der Unterschied lag aber darin, daß die zionistische Propaganda sich an die aktiveren und bewußteren Elemente wandte, die eine politiscbe und nicht eine lediglich individuelle Lösung suchten und ihnen eine falsche politische Lösung anbot. Darüberhinaus versuchte sie - gewissermaßen als Voraussetzung und Ziel ihrer Kampagne -, hartnäckig diese Juden davon abzuhalten, dem revolutionären Kampf ihrer Länder beizutreten.

Es gibt auch Ausnahmesituationen, in denen die Möglichkeit eines Kampfes der unterdrückten Minorität überhaupt nicht besteht und diese einer besonders extremen Gefahr ausgesetzt ist. In diesen Fällen ist die einzig menschliche Lösung die schnelle Organisierung der Auswanderung der unmittelbar gefährdeten Menschen in allen Ländern, die bereit sind, ihnen Asyl anzubieten. In einer solchen Situation befanden sich z.B. 1972 die Indienstämmigen in Uganda. In einer solchen Situation befanden sich die Juden in Deutschland und in anderen europäischen Ländern Ende der Dreißiger Jahre. Es war klar, daß, um diese Juden vor der Vernichtungsgefahr zu retten, es notwendig war, ihre Auswanderung nacb jedem sicheren Ort $\mathrm{zu}$ ermöglichen. Und zu diesem historischen Zeitpunkt zeigte der wahrlich grausame Zionismus (ohne Anführungsstriche) sein absolut zynisches Verhältnis zum Problem der Rettung der Juden. Die Führer des Zionismus zeigten Gleichguiltigkeit und sogar Feindseligkeit gegenüber der Auswanderung von Juden aus den gefährdeten Ländern in Orten außerhalb Palästinas. Der Zionismus zeigte deutlich, daß er im Prinzip nicht an der Rettung von Juden an sich, sondern nur an deren Rettung durch Emigration nach Palästina interessiert ist. Der Führer der Zionistischen Bewegung, Chaim Weizmann sagte: „Der Zionismus ist das ewige Leben, demgegenüber ist die Rettung Tausender von Juden lediglich Leben auf Zeit" (26).

Besonders erschütternd ist der Brief David Ben Gurions vom 17. Dezember 1938 an seinen Kollegen der Zionistischen Exekutive. Als Reaktion auf Versuche der Westmächte, verschiedene Notlösungen für das Problem der Juden Deutschlands $\mathrm{zu}$ finden, schreibt Ben Gurion: „Die gegenwärtige Judenfrage stellt sich heute anders, als in der Vergangenheit. Was jetzt den Juden in Deutschland geschieht, ist nicht das Ende, sondern der Anfang. Von den Handlungen Hitlers werden andere antisemitische Staaten lernen (...) Millionen Juden stehen jetzt vor der physischen Vernichtung. Das Flüchtlingsproblem wurde jetzt zu einer weltweiten und brennenden Frage, und England bemüht sich, das Flüchtlingsproblem und das Palästinaproblem auseinander zu halten. Das schreckliche Ausmaß des Flüchtlingsproblems erfordert eine rasche territoriale Lösung, und wenn Palästina keine Juden aufnimmt, so wird man ein anders Territorium suchen. Der Zionismus ist jetzt gefährdet, alle anderen territorialen Versuche, die zum Scheitern verurteilt sind, werden große Kapitalien erfordern, und wenn vor den Juden die Wahl stehen wird zwischen 
einerseits dem Flüchtlingsproblem und die Rettung der Juden aus Konzentrationslagern und andererseits der Hilfe für das nationale Museum in Palästina, dann wird das jüdische Mitleidsgefühl überwiegen und die ganze Kraft des Volkes wird der Hilfe für die Flüchtlinge in den verschiedenen Ländern zukommen. Der Zionismus wird von der Tagesordnung verschwinden und zwar nicht nur in der öffentlichen Meinung der Welt in England und in Amerika, sondern auch in der jüdischen öffentlichen Meinung. Wir riskieren das Leben des Zionismus, wenn wir es zulassen, daß das Flüchtlingsproblem und das Palästinaproblem voneinander getrennt werden" (27).

Nicht nur, daß Zionismus und Rettung von der Vernichtung bedrohten Juden nicht ein und das gleiche ist, sondern in einem kritischen, historischen Moment stand der Zionismus auf einer Position, die gegen die Rettung von Juden ausgerichtet war. Und hier müssen wir noch etwas hinzufügen: Es ist wahr, daß diejenigen Juden, die vor dem zweiten Weltkrieg an der zionistischen Auswanderung von Mittel- und Osteuropa teilnahmen, dadurch der Vernichtung durch den Faschismus entgingen. Aber der Versuch, aus dieser Tatsache eine sozialistische Legitimation für den Zionismus abzuleiten, ist nichts anderes, als Demagogie und moralische Erpressung.

Erstens, retteten sich viel mehr Juden ohne jeden Zionismus, ja sogar im Gegensatz zum Zionismus, sei es durch Auswanderung nach Amerika, sei es durch die Flucht ins Innere der Sowjetunion. Zweitens hing die Rettung der Juden in Palästina von der Tatsache ab, daß die deutsche Armee unter Rommel in Afrika nur bis Al-Alamein kam, ehe sie Palästina erobern konnte. Palästina lag ebenfalls auf der Route der faschistischen Eroberer. Hätte die Armee Rommels Palästina erobert und wäre bis Syrien gekommen, dann wäre das Schicksal der Juden in Palästina ohne Zweifel gleich dem ihrer Brüder in Polen gewesen. Keine ,magisch-mystische“" Kraft des Zionismus hätte dann die Juden der zionistischen Gemeinschaft vor den Nazis geschützt.

Nur einige wenige Zionisten waren bereit, die Unhal tbarkeit des zionistischen Axioms, nachdem man mit dem Zionismus aus der Weltgeschichte ,aussteigen " könnte und sich der Zionismus außerhalb des Prozeßes Faschismus-Antifaschismus befinden würde, zu erkennen. So sagte der Zionistenführer Yaakov Serubawel, im Januar 1945 vor dem Kongreß des „Weltverbandes Poalei Zion“ und rief damit heftigen Widerspruch hervor: „Ist es denn zulässig alles auf diese Katastrophe zu bauen? (die Vernichtung der europäischen Juden - A.d.V. ...). Und, daß wir in Palästina überlebt haben, ist das nicht ein Zufall? Stand Hitler nicht vor den Toren des Landes? Was wäre dann unsere Lage und unser Schicksal hier gewesen? Große

zit. nach Y. Elam, a.a.O., S. 125/26. Der historische Hintergrund war der Aufstand der Araber Palästinas gegen die britische Herrschaft, den Großbritannien nur mit Mühe unterdrücken konnte. Die britische Regierung wollte zu diesem Zeitpunkt die einheimische arabische Bevölkerung durch die Zulassung einer großen Welle zionistischer Kolonisation nicht allzu sehr verärgern, wobei sie von antizionistischen Juden unterstützt wird. 
Bevölkerungsteile bei uns und sicherlich die hier Anwesenden hätten sich zu verteidigen gewußt, so wie sich die Juden dort, in Warschau, zu verteidigen wußten. Hitler plante nicht nur die Diaspora, sondern das Judentum zu vernichten, alle sich überall befindlichen Juden. Wir haben uns durch Zufall gerettet" (28).

Denjenigen, die in der Judenvernichtung durch den deutschen Faschismus den „Beweis“ für die Ungültigkeit der marxistischen Auffassung zum jüdischen Problem und seine Lösung durch den gesellschaftlichen Kampf und die gesellschaftlichen Umwälzung erbringen und beiläufig auch die „Notwendigkeit“" des Zionismus suggerieren möchten, soll mit Isaac Deutscher geantwortet werden:

„Meiner Meinung nach bedeuten die tragischen Ereignisse während der Nazi-Ära weder die Ungültigkeitserklärung der klassischen marxistischen Analyse der Judenfrage, noch fordern sie eine Revision derselben ... Der klassische Marxismus rechnete mit einer gesünderen und normaleren Entwicklung unserer Zivilisation im allgemeinen, sozusagen mit einer zeitlich fortschreitenden Veränderung der kapitalistischen in einer sozialistischen Gesellschaft. Er rechnete nicht mit den hartnäckigen Überleben des Kapitalismus und seinen verderblichen Auswirkungen auf unsere Zivilisation im ganzen. Nichtdestotrotz wiederholten Marx, Engels, Rosa Luxemburg und Trotzki mehrmals, daß die Menschheit mit der Alternative: entweder internationaler Sozialismus oder Barbarismus, konfrontiert war - tertium non datur... Das europäische Judentum hat den Preis für das Überleben des Kapitalismus gezahlt, für den Erfolg des Kapitalismus in der Verteidigung gegen eine sozialistische Revolution.

Diese Tatsache ruft bestimmt nicht zu einer Revision der klassischen marxistischen Analyse auf - sie bestätigt sie eher" (29).

In der Tat gab es auch keinen wesentlichen Zusammenhang zwischen der Rettung von Juden im zweiten Weltkrieg und dem Zionismus. Was die Rettung von Juden in Palästina bewirkte, war die Tatsache, daß die Hitler'sche Kriegsmaschine zum Halten gebracht werden konnte. Die Juden wurden überall dort gerettet, wo der National-Sozialismus nicht hinkommen konnte. Die daraus zu ziehende historische Konsequenz ist, daß allein der weltweite Kampf gegen den Faschismus und die Reaktion eine wirksame Antwort auf den Antisemitismus ist. Diese Konsequenz steht im genauen Gegensatz zu jener, die sog. , linke" Zionisten ziehen. Daher muß ein weiterer Begründungszusammenhang der ,linken“" zionistischen Kritik am Antizionismus erörtert werden. Die Kritiker verurteilen die israelischen Antizionisten weil sie nicht darauf hinweisen, daß die Emigranten aus Ost- und Westeuropa „und in jüngster Zeit aus den arabischen Ländern“, nach Palästina/Israel wegen des Druckes des Antisemitismus und der Existenznot kamen; ,, die zionistische Ideologie spielte in den meisten Fällen keine oder höchstens eine sekundäre Rolle (. . .) für diese Menschen bedurfte es keines Druckes oder zionistische Propaganda, um sich zur Auswanderung nach Palästina zu entschließen" (30). 
Dazu ist folgendes zu sagen: Erstens will niemand die Tatsache bestreiten, daß der Zionismus Zigtausende von Menschen, die einen Ausweg aus der Not und der Unterdrückung suchten, - unter ihnen viele, die zunächst keine besonders begeisterten Zionisten waren - als menschlichen Rohstoff für das eigene Unternehmen benutzte. Andererseits ist jedoch die Behauptung, der Zionismus hätte auf diese Leute keinen besonderen Druck ausüben müssen, um sie zu einer Auswanderung nach Palästina/Israel zu bewegen, sehr entfernt von der Wahrheit. Als ein Beispiel soll die Affäre der Auswanderung der Juden Iraks Anfang der 50er Jahre ins Gedächtnis gerufen werden. Der Ablauf der Geschichte in allgemeinen Zügen: Im Jahre 1950 schloß die Zionistische Bewegung mit der reaktionären Regierung des Iraks ein geheimes Abkommen ab, nach dem die Emigration der Juden dieses Landes nach Israel betrieben werden sollte. Die irakische Regierung schloß unter anderem dieses Abkommen ab, weil sie daran ein finanzielles Interesse hatte: das Eigentum der ausgewanderten Juden sollte beschlagnahmt werden und in die Hände der Regierung übergehen. Sowohl die Zionisten als auch die Leute Nouri Saids (der damalige irakische Regierungschef) waren mit diesen Arrangement vollauf zufrieden. Das einzige Problem lag darin, daß die irakischen Juden selbst nicht mitspielen wollten. Nach ihrem Verständnis hatten sie keinerlei Grund, aus dem Irak nach Israel auszuwandern. Ihr Verhältnis zu den islamischen und christlichen Teilen der irakischen Bevölkerung war im allgemeinen harmonisch, so wie das Verhältnis dieser Teile der Bevölkerung den Juden gegenüber im allgemeinen normal und konfliktlos war. Da geschah etwas merkwürdiges: Einige Bomben explodierten an verschiedenen Orten, zum Beispiel in Bagdader Synagogen. Einige Juden fielen den Bomben zum Opfer. Als Resultat gerieten die irakischen Juden in Panik und binnen kurzer Zeit meldeten sich die meisten von ihnen zur Auswanderung nach Israel an. Nach einiger Zeit stellte sich ohne jeden Zweifel heraus, daß die Bombenleger Beauftragte der Zionistischen Bewegung waren, die Anweisungen ihrer Bewegung ausführten. Die Führer des ,grausamen“ Zionismus hatten also beschlossen, daß dort, wo es nicht genug Antisemitismus gibt, dieser gezielt erzeugt und/oder vorgetäuscht werden muß, um die Juden zu verängstigen, und sie zur Verwirklichung der zionistischen Lösung zu motivieren. Alle Einzelheiten dieser Affäre, die sich auf Aussagen irakischer Juden und einiger der „Helden“", der Bombenleger selbst stützen, wurden in Israel erst 15 Jahre später veröffentlicht. Viele heute in Israel lebenden Juden aus Irak, gefragt nach der Identität der damaligen Bombenleger geben in privaten Gesprächen offen zu: „Matnuáh“ - ,die Bewegung"“ - womit im hebräischen Sprachgebrauch die Zionistische Bewegung gemeint ist.

Dies ist nicht die einzige Affäre dieser Art. Jedoch wurden zu diesem Fall die Einzelheiten vollständig bekannt (31). 
Aus den obigen Erörterungen geht nun hervor, daß Zionismus und - Rettung-vonJuden vor Not und Antisemitismus keineswegs ein und dasselbe ist. Mehr noch: das spezifische am Zionismus ist nicht, daß er die Lösung der Frage der Juden allgemein durch deren Emigration herbeifuhren will. Das spezifische liegt vielmehr in der Forderung des Zionismus, daß die jüdische Emigration ausschließlich auf eine systematische Kolonisation Palästinas ausgerichtet sein muß, mit dem Ziel, dort einen exclusiv-jüdiscben Nationalstaat zu erricbten. Aus diesem Ziel resultieren zwangsläufig die Charakterzüge des ,zionistischen Unternehmens“ in Palästina. Die „,linken“ Zionisten erklären dazu, daß ,,es ein von Arabern bewohntes Land war, in das sie emigrierten, von allem Anfang an, die zweifellos häufig nicht erkannte oder verdrängte Tragik der jüdischen Einwanderung nach Palästina (war), aber wer darf von einer Volksgruppe - wer immer sie ist, und wann immer das war - die Bereitschaft zum kollektiven Selbstmord verlangen, solange die Möglichkeit zur Auswanderung besteht, selbst wenn das Zielland schon von anderen Menschen bewohnt ist" (32).

Für die Juden, die es wählten, sich von Not und Verfolgung durch private Emigration zum Beispiel in die USA zu retten, - und sie sind das vielfache von denen, die die zionistische Lösung wählten - war keinerlei Tragik darin, daß die USA bereits bewohnt war. Es kam ihnen gar nicht in den Sinn, daß, um sich selbst vor dem ,kollektiven Selbstmord“ zu retten, sie, die Nichtjuden aus den USA zu vertreiben hätten. Die "Tragik“ begann erst in dem Moment, als sich die zionistischen Siedler zum Ziel setzten, sich in Palästina nicht nur niederzulassen, sondern es von einem arabischen Land in einen exclusiv-jüdischen Nationalstaat zu verwandeln. Wir schrieben das Wort „Tragik“ in Anführungsstrichen, da die ,linken“ Apologeten des Zionismus es verwenden, um den Eindruck zu erwecken, hier handle es sich um irgendein grausames Spiel des blinden Schicksals und nicht um das Resultat von bewußten und gezieltem Handeln der Führer der zionistischen Kolonisatoren.

Chaim Weizmann, der Präsident der Zionistischen Organisation erklärte vor dem Pariser Friedenskongreß, im März 1919, die Ziele des Zionismus folgendermaßen:

„Mit der Gründung einer jüdischen nationalen Heimstätte, beabsichtigen wir in Palästina derlei Bedingungen zu schaffen, die uns ermöglichen sollen die jährliche Überführung von 50 - 60 Tausend Juden, die Entwicklung unserer Sprache, die Gründung unserer Schulen, Universitäten und anderen nationalen Institutionen und weiter ordnungsgemäß in dieser Richtung zu arbeiten bis schließlich Palästina so jüdisch sein wird wie Amerika amerikanisch und England englisch ist" (33). Und 
was sollte nun mit der arabischen Bevölkerung Palästinas passieren, die im arabischen Land Palästina die Mehrheit stellte? - Es ist falsch zu behaupten, diese Tatsache wäre ,häufig nicht erkannt oder verdrängt" worden. Einige prominente Zionisten sind da viel ehrlicher als mancher ihrer Apologeten; Menachem Ussischkin, Mitglied der Zionistischen Exekutive, berichtet über die geplante zionistische Lösung dessen, was im zionistischen Sprachgebrauch die ,arabische Frage" genannt wurde:

,Wir sind für ewig dazu verurteilt, eine kleine Insel im arabischen Meer zu bleiben; das heißt aber nicht, daß wir uns demütigen lassen und unterwerfen sollen. Wir müssen schweigen und nach Palästina gehen. Wir werden noch manche schwere Minute durchleben müssen. Geben wir aber nach Palästina, zebn um zebn, bundert um bundert, tausend um tausend, bunderttausende; und die arabische Frage ist gelöst" (34).

Die ,arabische Frage" ist für den Zionismus befriedigend ,gelöst", das arabische Volk Palästina wurde im eigenen Land zum Ausländer gemacht. „Tragik“?

Die territoriale Expansion des Zionismus die man an Hand der schon berühmt gew ordenen Landkarten Israels $(1947,1949,1967,1973)$ genau nachzeichnen kann, ist kein Zufall, kein historischer Unfall. Sie entspringt dem globalen Selbstverständnis der Zionistischen Bewegung, die zum einen den ausschließlich zionistischen Anspruch auf das Land Palästina - und natürlich nur für Juden - erhebt, während sie zum anderen glaubt die objektive Unvereinbarkeit des zionistischen Gebildes mit seiner arabischen Umgebung durch die von einer Ausweitung der Landesgrenzen sich ergebenden militär-strategischen und demographischen Vorteile begegnen zu können. Die Einverleibung arabischer Territorien unter zionistischen Herrschaft hat Geschichte und Methode. Im Jahre 1918 umfaßte die Bevölkerung Palästinas 599.000 Araber und 67.000 Juden, die jeweils 2 Mio ha bzw. 65.000 ha Boden besaßen. 1970 sind nur noch 86.000 ha des israelischen Bodens, d.h. ca. $4 \%$ in arabischen Händen (35). Mußte der Zionismus bis 1948 die Inbesitznahme des Bodens und seine Kolonisierung „Schritt um Schritt" durchführen, so konnte er nach Erlangung der staatlichen Hoheit sowohl die Ländereien und Dörfer der palästinensischen Flüchtlinge (in der israelischen juristischen Sprache ,Verlassenes Eigentum") übernehmen, als auch wesentliche Teile des Bodens der in Israel verbliebenen Araber, durch ihre administrative Verwandlung in „Geschlossene Militärgebiete" und ihre anschließende Beschlagnahmung, einverleiben. Mit solchen Mitteln wurde z.B. die seit den 50er Jahren forciert betriebenen „Judaisierung“ Galiläas bewerkstelligt (36). Die zionistische Bodenpolitik hat nichts dem Zufall überlassen. Wenn sie mit Unrecht, Vertreibung und viel Leiden für die Araber Palästinas verbunden war, so war dies keine „Panne“ sondern die logische Konsequenz

zit. nach: Der XII. Zionistenkongreß in Karlsbad vom 1. - 14.9.1921, Berlin 1922, S. 70

35

36

L. Gaspar, Histoire de la Palestine, Paris 1970, S. 104 u. S. 119

vgl. hierzu die ausführlich belegte Darstellung

in: Sabri Jeries/Eli Lobel, Die Araber in Israel, München 1970, S. 115 - 141 
aus der vom Zionismus bewußt und zielstrebig praktizierten Politik.

Wer aufmerksam die Selbstdarstellung der Zionisten verfolgt hat konnte feststellen, daß diese aus ihrem Herzen niemals eine „Mördergrube“ gemacht haben. In der Zeit, als die Grundlagen des Zionistischen Unternehmens in Palästina gesetzt wurden, konnte man die vorgesehene „Kontinuität“ schon schwarz auf weiß zu Gesicht bekommen. Bevor der Terminus „Kolonisation“ und „Kolonialismus“ allgemein in der Welt zu einem Schimpfwort wurde, benutzte es auch die Zionisti sche Bewegung um ihr Vorgehen in Palästina zu bezeichnen, sie sprach vor „Kolonisatzia“; der verdächtige Beigeschmack des Wortes bewog sie jedoch spätes die hebräischen Umschreibungen für den gleichen Begriff zu verwenden. Auf ihrem Gründungskongreß in Petach-Tikwa im Jahre 1919 verkündete die seit damals in der Zionistischen Bewegung führende, „linke“ Partei Ben Gurions, die Achduth Haawodá das Ziel der „Zionistischen Arbeiterbewegung in Palästina“ (sic): „Überführung des Bodens von Palästina, seiner Flïsse und seiner Naturschätze in den Besitz des gesamten jüdischen Volkes" (37). Ein klares Ziel ohne Zweifel, wußten die Zionisten doch ganz genau, daß ,unser Land nicht nur klein (ist), sondern zum. größten Teil im Besitz anderer" (38). Ein kompliziertes und verhängnisvolles Unternehmen, nach der übereinstimmenden Meinung von Befürwortern und Gegnern desselben, die eines ganz sicher wußten: Palästina war schon bevölkert, seine Umwandlung in ein ,jüdisches" Land würde auf Kosten der Einheimischen gehen müssen! Der zionistische Ökonom aus Jerusalem Alfred Bonné sagt: „Das Bodenproblem gehört zu den Fragen, die mit der Ausdehnung der jüdischen Kolonisation in den letzten Jahren eine besondere Aktualität und politische Bedeutung gewonnen haben. Wäre Palästina ein unbesiedeltes Land gewesen, oder hätten dort Verhältnisse wie in den kaum besiedelten kolonialen Territorien Australiens, Afrikas und Südamerikas geherrscht, so wäre die Bedeutung der Frage nicht über die Grenzen einer rein wirtschaftlichen hinausgegangen. Aber Palästina war, als die jüdische Kolonisationsbewegung einsetzte, ein besiedeltes Land und wies im Durchscbnitt sogar eine stärkere Bevölkerungsdicbte auf als die Nacbbarländer" (39); Yaakow Meiersohn, der bereits vorher zitiert worden ist, sagt 1920: „In Palästina gibt es überhaupt keinen unbesiedelten Boden; der Boden Palästinas ist besiedelt, jedoch nicht intensiv bearbeitet. Ich erkläre offen und entschieden, daß bisher in Palästina kein Stück Boden gekauft wurde, das nicht vorher von Arabern bearbeitet worden sei“ (40). Die Kommunistische Partei Palästinas sagte dazu: „Die Zionistische Bewegung mag nicht Ländereien kaufen, die vor der Bebauung noch der Trocknung bedürfen. Sie bevorzugt da schon den von den Fellachen langährig bebauten Boden (...) Erstens ist es wirtschaftlicher und gemeinnütziger, Kibbutzim auf bereits bebauten als auf unbebauten Ländereien $\mathrm{zu}$ errichten und zweitens

Ben Gurion, a.a.O., S. 117

38 vgl. die Rede von Saskin, Mitglied des Unterausschusses für Kolonisation in der Zionistischen Exekutive auf dem XII. Zionistenkongreß, Prot. a.a.O., S. 104

39 A. Bonné, Palästina, Land und Wirtschaft, Berlin 1935, S. 154/55

40 Y. Meiersohn, a.a.O., 
erfuillt man somit eine (zionistische A.d.V.) Pflicht: vom ,heiligen', jetzt durch jüdische Arbeiterhände, erlösten` Boden werden die Araber, die ,Goyim` vertrieben“" (41).

Niemand kann heute bestreiten, daß die Zionistische Bewegung Palästinas, die von 1920 bis Mitte der 60er Jabre unter der Führung Ben Gurions stand, etwas anderes beabsichtigte als für eine so zahlreich wie mögliche jüdische Majorität, ein so groß wie erreichbar - weitgehend ,araberreines" - Land . . . anzustreben. Ben Gurion schreibt: „An erster Stelle bin ich Zionist und strebe die Konzentrierung des jüdischen Volkes in seinem Land an; erst danach sehe ich die arabische Frage aufkommen“. Und weiter: „Wenn der zionistische Gedanke irgendeinen wirklichen Inhalt besitzt, so ist es der Inbalt vom Staat. Zionismus, das ist der Wille zum Staat der Juden der Drang zum Land Palästina und zur Errichtung der Landesregierung"; Und vier Jahre später, 1928 schreibt er: „Palästina für das jüdische Volk und Palästina für das arabische Volk ist nicht ein und dasselbe (...) wir würden uns betrügen, wenn wir sagten, daß es dasselbe wäre (. . ) Palästina ist für das jüdische Volk bestimmt und für diejenigen Araber, die dort leben" (42); $\mathrm{Zu}$ bemerken ist, daß Ben Gurion hier alle Juden der Welt meint und sie als Volk bezeichnet, während es in Palästina nicht einmal ein arabisches Volk gäbe, sondern lediglich „die Araber, die dort leben“" 1931 sagt er: „Ich habe ein arabisches Problem immer nur vom zionistischen Standpunkt aus gesehen, daß heißt, daß ich das Problem des jüdischen Volkes in Palästina lösen wollte, es in diesem Land sammeln wollte, um es $\mathrm{zu}$ einem freien Volk zu bilden, das in seinem Land lebt. Außerbalb dieses zionistischen Standpunktes gibt es in Palästina kein arabiscbes Problem, sondern nur ein jüdisches - wie übrigens überall" (43).

$\mathrm{Daß}$ der führende Flügel im Zionismus, nämlich die lautstarke „Arbeiterbewegung“ Kolonialismus unter dem Deckmantel von „Sozialismus“ betreibt mag zwar verwirren, aber die Tatsachen sprechen für sich. Für diejenigen, die nicht verstehen konnten wie Sozialismus mit Kolonialismus, Internationalismus mit Nationalismus, Enteignung und Verdrängung mit Klassenbrüderschaft zu vereinbaren sei erließen die „linken“ Zionisten schon 1912 ihren „Richterspruch": „Immer wenn wir einen Widerspruch zwischen dem nationalen und dem sozialistischen Prinzip vorfinden, neigt der Widerspruch dazu, auf das sozialistische Prinzip zugunsten der nationalen Tätigkeit $\mathrm{zu}$ verzichten. Wir akzeptieren den entgegengesetzten Versuch nicht, den Widerspruch durch den Verzicht auf die nationalen Interessen zugunsten der sozialistischen Idee $\mathrm{zu}$ lösen" (44). Durchschaut man die ,sozialistischen“ Beteuerungen des Zionismus, ihre Widersprüchlichkeit und Unhaltbarkeit, verliert die Zionistische Bewegung eines

zit. nach der Stellungnahme der Gewerkschaftsabteilung der PKP vom Oktober 1924, abgedruckt in: M. Offenberg, a.a.O., S. $336-337$

42 D. Ben Gurion, a.a.O., S. $299 / 300$, S. 275 u. S. 339

43 D. Ben Gurion, Wir und unsere Nachbarn, Jaffa 1931, S. 81/82 (hebr.)

44 Y. Ben Zwi in: Achaduth Nr. 16, Tel Aviv 1912 
ihrer wichtigsten propagandistischen Steckenpferde, die schon allzuviel subjektiv aufrichtige doch verworrene Sozialisten fur ein objektiv verwerfliches Kolonisationsund Unterdrückungsunternehmen einspannen und mißbrauchen konnte.

In der Tat ist es so, ob ,bürgerlicher" oder ,linker" Zionismus: Für die praktische Verwirklichung des zionistischen Unternehmen in Palästina, für die Folgen, die der zionistische „Aufbau" für die Araber Palästinas hatte, schlechthin für die objektive Konsequenz des zionistischen Werks im Lande Palästina, ist es ohne Bedeutung, wie man subjektiv ,den eigenen“ zionistischen Ansatz - und den „,der anderen“ - verstanden wissen möchte.

Hier liegt eindeutig geplante Politik vor. Bereits der Begründer des Zionismus, T. Herzl, schreibt in seinem Tagebuch am 12. Juni 1895:"6

„Mit dem Erwerb des Landes bringen wir sofort materielle Vorteile dem Land, das uns aufnimmt. Den privaten Boden in den Gebieten die uns gegeben werden, müssen wir nach und nach aus den Händen ihrer Eigentümer herausbekommen. Die armen Bewohner sind wir bemüht ohne Aufsehen zu erregen, durch Vergabe von Arbeit in den Transferländern, über die Grenze zu bringen. Jedoch in unserem eigenen Land geben wir ihnen keinerlei Arbeit (...). Es ist gut, wenn die Bodenbesitzer glauben, sie würden uns ausnutzen und für ihren Boden überhöhte Preise erhalten. Aber kein Boden wird zurückverkauft" (45).

Dies war und ist bis zum heutigen Tage die bewußte und geplante Politik des Zionismus: die ,arme Bevölkerung“ d.h. die Mehrheit der Araber im ,versprochenen Land" soll mit allen Mitteln aus dem Land ausgeschlossen werden. Im Jahre 1940 schrieb Josepf Weitz, damals der Chef der Kolonisationsabteilung der „Jewish Agency $^{\text {" }}$ in Palästina und damit der für die praktische Durchführung der zionistischen Kolonisation Verantwortliche in seinem Tagebuch:" Unter uns muß es klar sein, $d a ß$ es in diesem Land für zwei Völker keinen Platz gibt (. . .) Gemeinsam mit den Arabern werden wir nicht zu unserem Ziel gelangen, ein selbständiges Volk in diesem kleinen Land zu sein. Die einzige Lösung ist ein Palästina, zumindest ein West-Palästina (womit das ganze Gebiet westlich des Jordans gemeint ist, zu unterscheiden von „Ost-Palästina“, das das östliche transjordanische Gebiet meint. A.d.V.) ohne Araber (...) und es gibt keinen anderen Weg als die Araber von hier in die Nachbarländer zu überfuihren; alle zu überführen, es darf kein Dorf, kein einziger Stamm zurückbleiben (...) Für diesen Zweck wird man schon Gelder finden, viele Gelder wird man dafür auftreiben können. Und nur nach dieser Überführung wird dieses Land Millionen unserer Brüder aufnehmen können" (46).

Josepf Weitz selbst informiert uns in einem Artikel in der Tageszeitung „Davar" (offiziell das Organ der „Histadruth ${ }^{\text {s6 }}$ tatsächlich aber Sprachrohr der in Israel seit jeher herrschenden Mapai/Awodah-Partei ist) vom 29. Sept. 1967, daß dieser ausgezeichnete Plan, den er 27 Jahre zuvor in sein Tagebuch eintrug, nicht ein persönlicher Einfall von ihm war. Gleichzeitig unterstützten diesen Plan auch die 
wichtigsten Führer des Zionismus in Palästina, es wurde damit begonnen ,,vorzufühlen, wie man ihn praktisch realisieren könnte". In der Tat konnte ein großer Teil dieses Programms acht Jahre später verwirklicht werden: im Jahre 1946, als „,mit der Resulution der UNO über die Teilung des Landes in zwei Staaten zu unserem großen Glück (von uns hervorgehoben A.d.V.) der Befreiungskrieg ausbrach und in ihm ein doppeltes Wunder geschah: ein territorialer Sieg und die Flucht der Araber".

Es besteht kein Zweifel darüber, daß die Vertreibung der arabischen Fellachen von ihrem Boden sowie die Vertreibung der palästinensischen Araber aus ihrem Land keine „Tragik“ des blinden Schicksals, sondern das Ergebnis der bewußt geplanten zionistischen Politik war. Unter diesen Umständen muß die sich naiv gebärende Frage von ,linken“ Zionisten recht erstaunlich erscheinen: „Wie viele arabische Bauern (haben) in den Mandatsjahren 1920 - 1947/48, bevor sich die Araber gewaltsam gegen den Teilungsbeschluß der UN zur Wehr setzten, nun tatsächlich, trotz der Mandatsgesetzgebung zum Schutze der arabischen Bauern ihr Land verloren und keiner landwirtschaftlichen Beschäftigung mehr nachgehen können, und wie viele Araber (sind) in dieser Zeit aus den Nachbarländern nach Palästina eingewandert? “ (47)

Aus diesen Fragen können wir auf einige bezeichnenden Züge dieser Argumentation schließen. Erstens folgt daraus, daß die Vertreibung der arabischen Fellachen berechtigt war, nacbdem sich die Araber ,gewaltsam gegen den Teilungsbeschluß der UN zur Wehr setzten“. Solche Ansichten muß man mit schweigender Verachtung quittieren. Wir wollen nur ins Gedächtnis rufen, daß es bei all den heuchlerischen Apologeten des Kolonialismus in der ganzen Welt üblich ist, die massenhaften Vertreibungen der Kolonialvölker als eine gerechte Strafe dafür zu bezeichnen, daß diese bösen Eingeborenen es noch wagen, sich gewaltsam . . . ihrer massenhaften Vertreibung zu widersetzen. Zweitens wird daraus ersichtlich, daß die bekannten Absichten des Zionismus, wie sie in den oben zitierten Stellen sowie in vielen anderen Dokumenten zum Ausdruck kommen und die bekannten historischen Tatsachen, bewußt ignoriert werden sollen und stattdessen weißgemacht werden soll, der Zionismus hätte bis 1948 nicht arabische Fellachen im großen Maßstab vertrieben. Die Wahrheit sieht aber ganz anders aus.

Man kann ohne weiteres Beispiele von massenhaften Vertreibungen von arabischen Fellachen als Resultat der zionistischen Kolonisation anführen. Viele ereigneten sich bereits vor Gründung des zionistischen States und dauerten während der ganzen Zeit des britischen Mandats, also bis 1948, an.

Diese Fragen, ,linker" Zionisten sollen Glauben machen, der britische Imperialismus hätte - durch die Mandatsregierung - irgendeinen effektiven Schutz vor Vertreibung gegeben. Auch dies ist nicht wahr. In diesem Zusammenhang zitieren wir hier aus den Erinnerungen des jüdisch-englischen Zionisten M. Hyamson, der während der ersten Hälfte der Mandatszeit als hoher Regierungs- 
beamter in Palästina tätig war. M. Hyamson berichtet über den ersten Versuch, der Anfang der 20er Jahre unternommen wurde, arabische Pächter vor der Vertreibung zu schützen: „Die Notwendigkeit (dieser Bestimmungen) wurde dringend, weil jüdische Agenturen von (arabischen) Großgrundbesitzern, die in Paris, Beirut oder Kairo saßen, verhältnismäßig viele Ländereien kauften, wobei die moralischen wenn nicht die gesetzlichen - Rechte der Pächter, die seit jeher auf ihren Boden ansäßig waren, ignoriert wurden. Nach der neuen Gesetzgebung wurde die Übereignung von Ländereien verboten, wenn nicht die Interessen des Pächters dadurch sichergestellt wurden, daß $\mathrm{ihm}$ genug Boden zur Sicherstellung seiner eigenen Existenz sowie die seiner Familie, gelassen wurde. Dies aber stand im Widerspruch sowohl zu den Interessen der Verkäufer als auch zu den der Käufer. Die Käufer waren bereit, hohe, über das übliche hinausgehende Preise zu zahlen, verlangten jedoch, daß der Boden zur Besiedlung frei sein muß. Die Verkäufer, die keinerlei lokalen Interessen am Ort selbst hatten, waren natürlich gierig, so teuer wie möglich zu verkaufen. Sehr rasch fanden sie einen Weg, das Gesetz mit Hilfe einer kleinen Zahlung zu umgehen. Sie fanden Verbündete und deren Hilfe bei den Geldverleihern, bei denen die meisten Pächter hoch verschuldet waren. Sie nutzten diesen Weg, um die Pächter durch Überreden und durch Zahlung zu veranlassen, den Boden zu verlassen, noch bevor er übereignet wurde. Als es dann zur Übereignung kam, waren keine Pächter mehr da, für die man zu sorgen hätte. So waren alle für immer zufrieden; die Verkäufer, die Käufer und verständlicherweise auch die Geldverleiher, die Pächter allerdings nur kurzfristig." Die Pächter waren nur kurzfristig zufrieden, weil die „Entschädigung“, die sie vom Grundbesitzer bekamen, sehr klein war; sie reichte kaum aus, um ihre Schulden bei den Geldverleihern zu begleichen. Darüberhinaus „begegneten" wie Hyamson feststellt, jene Fellachen und Pächter, die zum Verlassen ihrer Ländereien gezwungen wurden, ,einer Weigerung, sie in den meisten im Land neu entwickelten Betriebe zu beschäftigen“. Diese Betriebe waren zionistisch und der Zionismus weigerte sich prinzipiell, arabische Arbeiter zu beschäftigen. Hyamson erzählt weiter, daß ,im Jahre 1929 eine neue Bestimmung erlassen wurde, die jedoch den Pächter noch weniger schützte (. . ), praktisch stellte sie eine Legalisierung der bereits etablierten Gewohnheiten dar" (ebenda). Zwei Jahre später ,begannen wieder Bodenkäufe in einem großen Ausmaß und das erwartete Problem der landlosen Araber stand wieder an der Spitze der Tagesordnung". Dieses Problem verursachte Unruhen und zwang die Mandatsregierung, neue Bestimmungen zu erlassen. Jedoch auch die neuen Bestimmungen aus dem Jahre 1931 gewährten den Pächtern keinen effektiven Schutz, denn ,diejenigen Grundbesitzer, die die Möglichkeit bewahren wollten, ihren Boden zu ihnen genehmen Preisen verkaufen zu können, die Absichten des Gesetzes immer noch umgehen konnten". Dieser Zustand dauerte bis zum Ende der Mandatszeit an (48).

Wir zitierten nur einen geringen Teil aus der interessanten Abhandlung Hymsons zu diesem Thema. Aus den obigen Stellen sowie aus der gesamten 
Abhandlung geht klar hervor, dał das Problem derjenigen Pächter, die die Grundlage ihres Lebensunterhalts, d.h. den Boden, den sie und ihre Vorväter seit Generationen bestellten, durch die zionistische Kolonisation verloren; ein äußerst ernstes Problem darstellte und daß die Zahl der Betroffenen groß war. Gleichfalls ist es klar, daß die Erlasse der Mandatsregierung die Pächter nicht wirksam gegen das auf gemeinsame Interessen beruhende Komplott der zionistischen Institutionen, der Großgrundbesitzer und Geldverleiher schützen konnten.

Nur ein Beispiel:

Die 8.000 Fellachen aus 22 Dörfern, die durch den Landverkauf der Großgrundbesitzerfamilie Ssursuks an die Zionisten, Anfang der 20er Jahren, ihr Land verloren hatten, erhielten von der zionistischen Organisation genau 1/2 Pfund pro Kopf (49).

Um die Folgen der zionistischen Kolonisation zu verharmlosen, wird von zionistischer Seite oft darauf hingewiesen, daß seinerzeit nur ,insgesamt 664 Entschädigungsforderungen" von arabischen Bauern gestellt wurden. Hierbei wird neben der Tatsache, daß die Möglichkeit der sog. und unverhältnismäßig kleinen „Entschädigung“ möglichst wenig publik gemacht wurde, unterschlagen, daß eine Reihe der enteigneten Bauern von vornherein von der Möglichkeit der Beanspruchung einer ,Entschädigung“" ausgeschlossen waren:

- Bauern, die im Anschluß an Verkäufe an Nicht-Juden vertrieben wurden. (Hierbei handelt es sich um Verkäufe an arabischen Vermittler und Wucherer, die das Land an die Bodenerwerbsabteilung der Zionistischen Organisation weiterverkauften).

- Bauern, die nicht als Pächter einzuordnen sind; Landarbeiter und Bauern, die nur einen Teil ihres Landes verkauft haben.

- Bauern, die ihre Pächterrechte nicht dokumentarisch nachweisen konnten (sehr viele!)

- Bauern, die nach dem Verkauf anderes Land zugewiesen bekamen, selbst wenn dieses Land nicht landwirtschaftlich nutzbar war.

- Bauern, die nach der Vertreibung einen anderen Arbeitsplatz gefunden hatten.

Damit war die Einstufungsdefinition als "landloser Araber" zugunsten des Zionismus auf eine kleine Gruppe beschränkt (50).

Im Zeitraum 1920 - 1936, die Zeit der Errichtung der Grundlagen des zionistischen Unternehmens in Palästina in Stadt und Land, setzte eine verstärkte „Landflucht" der besitzlosen Bauern ein - eine Flucht, die richtig zu verstehen ist: nicht ,von" dem Land, sondern eine Wanderung, nachdem der Bauer sein Land verloren hatte. Die arabische städtische Bevölkerung Palästinas wuchs von 194.000 im Jahre 1922 auf 298.000 im Jahre 1936.

Die arabische Landlosigkeit traf nun in den von den Zionisten beherrschten

50 vgl. A. Survey of Palestine hrsg. v. Palestine Government, vol. I, S. 296 und Palestine Royal Commission Report 1937, S. 239/40 
Städten auf steigende Arbeitslosigkeit, die sich aus dem Primat der ,hebräischen Arbeit" und dem Boykott der ,,arabischen Arbeit" ergab. Aber zurück zur Tatsache, daß die Fellachen meist schon vor der Übereignung und durch die Verkäufer (im vorsätzlichen Einvernehmen mit den Käufern) vertrieben wurden. Diese Tat* sache ermöglichte es dem Zionismus, wie Pontius Pilatus seinerzeit, seine Unschuld zu beteuern und zu behaupten, es sei nicht er, der die Vertreibung der Fellachen herbeiführte. Es gibt jedoch auch genügend Beispiele von Fällen, bei denen sich die zionistischen Kolonisatoren an der Vertreibung der einheimischen Fellachen wie in Al-Fule (heute Afula) Ende 1924 oder Wadi al-Hawarith (heute Emek Hefer) in den Jahren 1931 - 35 tatkräftig und in Zusammenarbeit mit der britischen Polizei beteiligten.

Bis zum heutigen Tage wird von den Propagandisten des Zionismus die Behauptung weiter verbreitet, daß die zionistischen Institutionen (zumindest bis 1948) in den meisten Fällen ,menschenleere Ländereien“ übereignet bekamen, so daß der Zionismus für die Vertreibung der Massen der Fellachen nicht verantwortlich sei. Formal gesehen und angewendet auf die passenden Fälle liegt hier keine Lüge vor, sondern eigentlich eine Halbwahrheit, die schlimmer als eine Lüge ist; denn die zionistischen Propagandisten verschweigen, daß um die Gesetze, die zum Schutz der Fellachen erlassen wurden, zu umgehen, die zionistischen Institutionen von den Verkäufern verlangten, daß diese vor dem Vollzug des Verkaufs selbst ihre Pächter vertreiben sollten.

Nebenbei bemerkt sehen wir hier, wieweit eine weitere Behauptung der „linken“ Zionisten von der Wahrheit entfernt ist. Nämlich die, nach welcher „es nicht die armen Fellachen, sondern die Großgrundbesitzer waren, die aus sehr klassenbewußten Gründen die jüdische Einwanderung und die dadurch befürchtete ,Ansteckung ihrer Fellachen mit aus Europa importierten sozialen Ideen ablehnten“. Erstens waren die ,,sozialen Ideen“ die der Zionismus aus Europa brachte, für den exclusiv-jüdischen Gebrauch bestimmt. Alle Institutionen der organisierten Arbeit und des gemeinschaftlichen Lebens waren keineswegs für Araber bestimmt. Auch propagierte der Zionismus niemals irgendwelche fortschrittliche sozialen Ideen unter den Fellachen. Im Gegenteil: der Zionismus war wie wir oben sahen, der objektive Bündnispartner der Großgrundbesitzer. Dies ist die einzige soziale Klasse der arabischen Gesellschaft, die durch die zionistische Kolonisation Vorteile genoß, und zwar indem sie für ihre Ländereien Preise bekamen, die höher waren, als vor der Kolonisation. Die Fellachen waren in der Tat das Opfer einer Allianz zwischen dem Zionismus und dem Großgrundbesitzer und Geldverleiher. Es ist wahr, daß, um ihre wahren Interessen und Absichten zu verschleiern, die Großgrundbesitzer manchmal eine scharfe verbale Kampagne gegen den Zionismus veranstalteten. Aber das waren nur Worte. Die wahren Opfer des Zionismus waren die Fellachen - diejenigen, welche bereits vertrieben waren und die, die unter der Vertreibungsgefahr noch lebten.

Es sei hier bemerkt, daß die Art und Weise der Vertreibung (die zumeist verschleiert und vertuscht wurde, um das Gesetz zu umgehen) sowie das Fehlen einer ordentlichen Bodenerhebung, eines Grundbuches, die Registrierung der Eigen- 
tums- und Nutzungsrechte des Bodens die Ursache dafür sind, daß es bis heute unmöglich ist, genaue Angaben über das Ausmaß der Vertreibung zu machen. Es besteht kein Zweifel daran, daß es viele Tausende gewesen sein müssen, jedoch müßte die genaue Zahl noch durch eine ernste Forschungsarbeit ermittelt werden. Die Frage, wieviele Fellachen ihren Boden durch die zionistische Kolonisation nun verloren hätten, kann also nur mit diesem pauschalen Hinweis beantwortet werden.

Wir zitieren hierzu noch aus der Rede Mosche Dayans vor den Studenten der Technischen Hochschule in Haifa (,Technion"), wie sie die israelische Tageszeitung „Ha'aretz Arabern bewohnt war und errichteten hier einen hebräischen, d.h. einen jüdischen Staat. In großen Gebieten kauften wir die Ländereien von den Arabern ab. Jüdische Dörfer entstanden an der Stelle arabischer Dörfer. Ihr kennt nicht einmal die Namen dieser Dörfer, und ich werfe euch dies auch nicht vor, da die entsprechenden Erdkundebücher nicht mehr existieren. Nicht nur die Bücher existieren nicht, auch die Dörfer gibt es nicht mehr. Hahalal entstand an der Stelle von Mah'lul, Gevat an der Stelle von Jibta, Sarid anstelle von Chanips und Kefar Yehoschua an der Stelle von Tel-Schaman. Nicht eine Ortschaft wurde hier im Lande an einer Stelle aufgebaut, an der es zuvor nicht eine arabische Bevölkerung gegeben hätte". In der Tat sprechen die Berufsgeneräle des Zionismus oft deutlicher und offener als manche ihrer „linken“ Apologeten. Die koloniale Besiedlung eines Landes und die daraus folgende Vertreibung und Unterdrückung seiner einheimischen Bewohner, das Ganze noch mit dem propagandistischen Ziel einer angeblichen ,fortschrittlichen“" Gesellschaft, die nach Meinung der als ,,sozialistisch“ verkleideten Zionisten in Palästina entstehen sollte, ist nicht bloß Heuchelei sondern auch die theoretische und praktische Prostitution der verbal vertretenen revolutionären Theorie.

Die erste systematische Erhebungsarbeit auf dem Gebiet der Erforschung des Ausmaßes der Zerstörung, die der Zionismus und seine Kolonisation an der ursprünglichen palästinensischen Gesellschaft angerichtet hat, die am 15. Februar 1973 vom Vorsitzenden der israelischen Sektion der Liga für Menschenrechte, Prof. Israel Schahak vorgelegt wurde, enthält eine komplette Liste derjenigen arabischen Ortschaften Palästinas, die bis 1948 bestanden, und die man heute vergeblich suchen würde. Sie gibt es nicht mehr. Ihre Anzahl: 385 - in Worten Dreihundertfünundachtzig.

Aus einigen der obigen Zitate ging hervor, daß es ein Teil der zionistischen Vertreibungspolitik war, auf die Araber durch ihre Nichtbeschäftigung stets Druck auszuüben. An diesem Punkt fühlen die ,linken“ Zionisten sich etwas unbequem . . . aber nur für einen Augenblick. Sie räumen zwar ein, daß die Verdrängung von arabischen Arbeitern von ihren Arbeitsplätzen zu den Dingen gehören, die ,auf uns Europäer abstoßend wirken". Jedoch fordern sie die Leser noch im gleichen Atemzug auf, sich von solchen barmherzigen, weichen, anscheinend spezifisch europäischen „Vorurteilen“ freizumachen. Ihr versteht wohl, die arabischen Arbeiter mußten verschwinden, ,,um diese(jüdischen) Arbeiter vor dem Verhungern zu bewahren, da es jüdischen Arbeitern einfach nicht möglich war, bei gleichem Lohn, wie er den arabischen Arbeitern gezahlt wurde, das nackte Existenzminimum 
zu erreichen". Man muß also verzeihen: die jüdischen Arbeiter hatten einen europäischen Magen, der größer war, als der ihrer arabischen Klassenbrüder.

Nach dieser glänzenden Argumentation schleicht sich doch wieder ein unwohles Gefühl ein, und es wird zugegeben, daß vielleicht „ein auch für die Araber günstigerer Modus zur Lösung dieser Frage hätte gefunden werden können. Man hätte zum Beispiel nur die tatsächlichen Verhältnisse - die arabischen Bauern verkauften unbehindert ihre Erzeugnisse zu billigeren Preisen auch in jüdischen Städten - irgendwie institutionalisieren müssen und manches Porzellan wäre nicht zerbrochen worden".

Dieser Entschuldigungsversuch scheitert jedoch gleich zweifach: Es wird eine Unwahrheit und eine Unsinnigkeit zugleich behauptet. Es ist eine Unwahrheit, daß die zionistischen Institutionen den Verkauf von Produkten durch die arabischen Fellachen nicht systematisch störten und bebinderten und zwar nicht nur durch Propaganda, sondern auch mit Hilfe wirksamerer "Überzeugungs"-Methoden. (So berichtet zum Beispiel der zionistische Führer David Hakohen in der Beilage der Zeitung „Ha'aretz" vom 15. Nov. 1968, wie er und seine Kollegen von Arabern angebotenen Tomaten mit Petroleum übergossen und Eier zerbrachen). Ebenfalls ist der Entschuldigungsversuch von Grund auf unsinnig, weil der einzige ,Modus" zur Lösung des Problems, der vermieden hätte, daß ,manches Porzellan nicht zerbrochen worden wäre“, darin besteht, daß der Zionismus sein Hauptziel aufgegeben hätte. Vom Standpunkt des zionistischen Zieles: - die Umwandlung Palästinas, was ein arabisches Land war, in einen ,jüdischen“ Nationalstaat - war die Anwesenheit der Araber ein Hindernis, das beseitigt werden mußte. Ein für die Verwirklichung dieses Zieles zwangsläufiger Weg war, wie alle Zionisten seit Herzl erkannten, den Arabern jede Beschäftigung vorzuenthalten.

Die Kontinuität in der Politik der „Zionisierung“ und gleichzeitiger „Entarabisierung" Palästinas hat sich in ihrem Kern nicht geändert. Im Gegenteil. Die im Juni-Krieg 1967 eroberten arabischen Gebiete gaben Israel die Möglichkeit mehr als 50 zusätzliche ,zivile“ und militärische Niederlassungen dort zu errichten und viele tausende von Arabern z.T. zum zweiten Mal in 20 Jahren zu vertreiben. Die „,wegweisenden“ Worte Mosche Hayans sprechen es aus: „Im Laufe der letzten 100 Jahre hat unser Volk einen nationalen und staatlichen Aufbauprozeß, durch Expansion, durch das Erreichen von mehr Juden, Niederlassungen und Kolonisation um hier die Grenzen auszuweiten, durchgemacht. Es darf keinen Juden geben der sagt, dies sei das Ende dieses Prozesses. Es darf keinen Juden geben, der sagt wir seien am Ende des Weges angelangt". (Fußnote: General Mosche Dayan, in Maariv, Tel Aviv 7. Juli 1968)

In der Tat scheinen aber nach Oktober 1973 die Konturen einer Eindämmung der zionistischen Marschroute sichtbar. Anknüpfend an unserer Einleitung weisen wir darauf hin, daß der Zionismus, zum ersten Mal in seiner Geschichte unmittelbar mit der Aussicht konfrontiert ist, territoriale, militär-strategische und kolonisatorische Errungenschaften wieder abgeben zu müssen. So begrenzt dieser Vorgang zunächst anmuten kann, er rüttelt am Kern Israels, ein Staat der insgesamt ein riesiges „fait accompli“" ist. Es ist jedoch nicht wahrscheinlich, daß auch ein 
zionistisches „Rumpf“-Israel, in den Grenzen vom 4. Juni 1967 ,plus Korrekturen“ einer friedvollen und konfliktlosen Koexistenz mit seiner arabischen Umgebung auf Dauer entgegen sehen kann. Der Nahostkonflikt ist kein bloßer „Grenzkonflikt ${ }^{6 \text {. }}$. Die Ursache für den historischen Konflikt zwischen dem Staate Israel, in seiner jetzigen Fassung einerseits und den Arabern andererseits, ist die Existenz und die Wirkung des Zionismus. Wer sich aufrichtig um die Zukunft von Israelis und Arabern im Nahen Osten bemüht, macht sich darüber Gedanken.

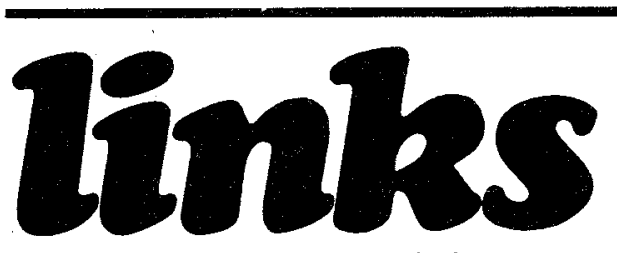

Sozialistische Zeitung

bringt monatlich auf ca. 24 Seiten Beiträge zur sozialistischen Theorie und Strategie, Aktionsmodelle, Berichte aus der Linken international. "links" ist eine Zeitung für Theorie der Praxis und für Praxis der Theorie.

Preis für das Finzelheft DM 1,50 Jahresabonnement DM 21,-- incl. Versand Probeexemplare und Bestellungen bei Sozialistisches Büro + Verlag $2000 \mathrm{GmbH}$ 605 Offenbach 4, Postfach 591 .

\section{J U N I 75}

Cravos: Der revolutionäre Prozeß in Portugal

R.B. Wurm: Verlage

Uta Claar: Berufsverbote

SB-Gruß nach Vietnam

A. Blechschmidt: Weltwirtschaftskrise Armanski u.a.: Krise in USA

\section{JULI/AUGUST ' 75}

O. Negt: Erfahrung, Emanzipation und Organisation

D.Horster $/$. Perels: Ernst Bloch

A. Buro: NATO und Portugal

W. Müller-Jentsch: Betriebsratswahlen

S. Berg: Einzelkämpfer in der Provinz

S E P T E M B E R ' 75

E. Altvater: Okonomische Krise und politische Disziplinierung

W.-D. Narr: Zum Berufsverbot

Cravos: Die Rolle der PS in Portugal

Schulstreik gegen 'SparmaBnahmen'

E. Wedekind: Kritik des SB-Schwerpunkts Betriebsarbeit

Jonas/Ludwig: Autonomie und Widerstand 